\title{
Relating the Effect of HPA Axis to the Emotional State of Bipolar II Disorder Patient
}

\author{
U. E. Michael ${ }^{1}$ and M. O. Oyesanya ${ }^{2}$ \\ ${ }^{1}$ Alex Ekwueme Federal University, Ikwo, ${ }^{2}$ University of Nigeria, Nsukka \\ Michael.uchenna@funai.edu.ng.mosesoyesanya@unn.edu.ng
}

Abstract

Using analytic approach we study the effect of HPA axis secretions to the emotional variation of bipolar II disorder patient. Modified Duffing - Van der Pol oscillator was used to model the emotional variation, that was solved analytically using multiple scale perturbation to obtain an asymptotic solution. The solution was graphed to understand the effect of the variation of the cortisol to the oscillator. It was observed that the increase or decrease of the HPA hormone from the basal level in the body system, affects the mood variation of bipolar II disorder patients.

Keywords: HPA axis, Bipolar II disorder, perturbation, cortisol

\subsection{INTRODUCTION}

The hypothalamic - pituitary - adrenal (HPA) axis helps to maintain basal and stress related homeostasis of central nervous system (CNS), cardiovascular, metabolic and immune functions [58]. Detailed dynamics of the HPA axis is complex, depending on the individual metabolic load of an organism, its current status and environmental impacts [59]. Cortisol, the HPA axis arch hormone in humans, exhibits complex dynamic behavior with two notable frequencies: ultradian oscillations, with a period of $20-120 \mathrm{~min}[60,61]$ superimposed on circadian oscillations, with a period of about 24 hours. There are many literatures that establish the importance of circadian rhythms in the functionality of HPA axis; recent experimental [62 - 64] and theoretical [65 - 67] results offer notable evidence to support the inherent roles of ultradian oscillatory dynamics of HPA hormones levels for normal physiology. Since the ultradian and circadian oscillations have disparity in their time scale, it will culminate that their effects result to disparate biological realms.

In the absence of an inherent oscillatory mode, it is difficult to attribute a role to HPA axis endogenous dynamics in the creation or amplification of circadian or ultradian cycles [68]. Regardless, circadian rhythms of hormones are synchronized by environmental factors such as light, they are generated by an endogenous system called circadian clock. The widely accepted understanding of the system is that the suprachiasmatic nucleus ( $\mathrm{SCN}$ ) of the hypothalamus controls the circadian system, but it is not limited to SCN [69]. SCN coordinates the regulation of cellular clocks because circadian rhythm exists even in the organ level by sending hormonal and electrical signals [70]. Originally, it was believed that the pulsatile release of CRH from the hypothalamus creates ultradian oscillations. But blocking the effect of $\mathrm{CRH}$ on the pituitary did not eliminate the pulsatile release of ACTH (71). Hence it is agreed that multiple and redundant mechanisms regulate the ultradian oscillations $[68,71]$.

Changes in the detailed dynamics of the HPA axis emerge routinely while the axis copes with a myriad of external stimuli [67]. Stress and some ailments are associated with short or long - term perturbation of the HPA dynamics. These factors change the amplitude and /or frequency of the basal HPA hormones discharge that results to many health condition, which includes bipolar disorder, Cushing's syndrome, hypertension, visceral obesity, diabetes, osteoporosis [60, 72]. 
Cortisol the arch - hormone of HPA axis, when elevated is associated with depression which also inhibits the production of thyroid stimulating hormone (TSH): [Thyroxin $\left(\mathrm{T}_{4}\right)$ and triiodothyronine $\left(\mathrm{T}_{3}\right)$ ]. Elevated cortisol levels are potentially related to reduced feedback inhibition of the HPA axis while low levels of cortisol are associated with increased feedback inhibition of the HPA system [73]. Besides the changes in the level of cortisol, alterations in the frequency of the ultardian oscillation have noticed in some patient with depression [68]. The contextual model of the axis dynamics is significant as to curb this lag in frequency alteration.

Purpose of this study is to underpin the effect of circadian oscillations of cortisol as it relates to its effect to mood variation which is evidential in bipolar disorder patient. In addition, we will consider the change of emotional state of bipolar II disorder patient with respect to the quantity of cortisol in the bloodstream.

\subsection{FORMULATION OF THE MODEL}

For the purpose of formulating a mathematical model of bipolar II disorder, some assumptions are pertinent. There is a periodicity that governs the utradian and circadian oscillation which induces same for hypomanic and depressive episodes; that have rapid cycling when the patient is treated with only antidepressant [1]. The amount of cortisol in the system of a patient has great effect to the emotional state of the patient; either hypomanic or depressive episodes, hence endocrine dysfunctionality corresponds to bipolar II disorder. Additionally, the bipolar II patient has mood swings that oscillate; hence the formation of limit cycle is inevitable. We can model the mood variations of a bipolar patient with a negatively damped harmonic oscillator and show the effect of psychosocial therapy on the patient. Our model is a special form of Duffing - Van der Pol oscillator

$$
\frac{d^{2} u}{d t^{2}}+\delta \frac{d u}{d t}+w^{2} u=f\left(u^{\prime}, u, x, t\right)
$$

where $u$ is the emotional state of the patient, $u^{\prime}$ is the rate of mood changes between hypomania and severe depression, $\delta$ is the damping coefficient, $w$ is the natural frequency of the oscillator. The solution of (1) often gives unbounded oscillations which is a major drawback of such a model. $f\left(u, u^{\prime}, x, t\right)$ in (1) represents the medication given to the patient, which we define a

$$
f\left(u^{\prime}, u, x, t\right)=\alpha u^{2}+\beta u^{3}-\varepsilon F \frac{d^{2} \bar{u}}{d x^{2}}
$$

where $\alpha, \beta$ are parameters that stabilize the emotional state of the patient, $F$ is the resultant external and internal force that induces mood swing by changing the level of cortisol in the system or otherwise, $\varepsilon$ is taken to be a bookkeeping device and $\bar{u}$ is the initial emotional state of patient at the point of observation initialization. Combining (1) and (2) we get

$$
\frac{d^{2} u}{d t^{2}}+\delta \frac{d u}{d t}+w^{2} u-\alpha u^{2}-\beta u^{3}=-\varepsilon F \frac{d^{2} \bar{u}}{d x} .
$$

Considering the emotional state of the patient with respect to the amount of cortisol in the blood; we use the Van der Pol oscillator to represent an untreated bipolar patient

$$
\frac{d^{2} u}{d x^{2}}+\delta \frac{d u}{d x}+2 \lambda u=0
$$

where $2 \lambda$ is the natural frequency on which the arch - hormone of HPA axis is released into the bloodstream. The central neuroendocrine system; HPA axis has a feedback mechanism that mediate on the functionality of the hypothalamus, pituitary gland and adrenal gland, hence we assume $\delta=0$, therefore 


$$
\frac{d^{2} u}{d x^{2}}+2 \lambda u=0
$$

Differentiating (5) twice and applying the treatment as an autonomous forcing function $f\left(u, u^{\prime}, x, t\right)$ we obtain

$$
\frac{d^{4} u}{d x^{4}}+2 \lambda \frac{d^{2} u}{d x^{2}}=f\left(u^{\prime}, u, x, t\right)
$$

On coupling (3) and (6) we obtain that

$$
\frac{d^{2} u}{d t^{2}}+\delta \frac{d u}{d t}+\frac{d^{4} u}{d x^{4}}+2 \lambda \frac{d^{2} u}{d x^{2}}+w^{2} u-\alpha u^{2}-\beta u^{3}=-2 \varepsilon F \frac{d^{2} \bar{u}}{d x^{2}}
$$

where $\bar{u}=a_{m} \sin m x ; a_{m}$ is the initial amplitude of the emotional state and $m$ is the frequency.

\subsection{Analytical Solution}

By using perturbation method [74], we can obtain information regarding the limit cycles of (7). Let

$$
0 \leq x \leq \pi, 0 \prec \delta \square 1 .
$$

Let

$$
u(x, t)=U(x, \hat{t}, \tau, \varepsilon \delta)
$$

We assume the following homogenous initial conditions

$$
u(x, 0)=u^{\prime}(x, 0)=0
$$

and the boundary condition

$$
u(0, t)=\frac{d^{2} u(0, t)}{d x^{2}} \text { at } x=0, \pi .
$$

We introduce a slow time scales, such that

$$
\tau=\delta t
$$

and

$$
\hat{t}=t+\frac{1}{\delta}\left(\varepsilon \mu_{1}+\varepsilon^{2} \mu_{2}+\varepsilon^{3} \mu_{3}+\cdots\right)
$$

where $\mu_{i}=\mu_{i}(\tau), \mu_{i}(0)=0, i \in \square$.

Thus we get

$$
\frac{d u}{d t}=U^{\prime}=\frac{\partial U}{\partial \hat{t}}+\left(\varepsilon \mu_{1}^{\prime}(\tau)+\varepsilon^{2} \mu_{2}^{\prime}(\tau)+\varepsilon^{3} \mu_{3}^{\prime}(\tau)+\cdots\right) \frac{\partial U}{\partial \hat{t}}+\delta \frac{\partial U}{\partial \tau}
$$




$$
\begin{aligned}
\frac{d^{2} U}{d \hat{t}^{2}}= & \frac{\partial^{2} U}{\partial \hat{t}^{2}}+2\left(\varepsilon \mu_{1}^{\prime}(\tau)+\varepsilon^{2} \mu_{2}^{\prime}(\tau)+\varepsilon^{3} \mu_{3}^{\prime}(\tau)+\cdots\right) \frac{\partial^{2} U}{\partial \hat{t}^{2}} \\
& +\left(\varepsilon \mu_{1}^{\prime}(\tau)+\varepsilon^{2} \mu_{2}^{\prime}(\tau)+\cdots\right)^{2} \frac{\partial^{2} U}{\partial \hat{t}^{2}}+2\left(\varepsilon \mu_{1}^{\prime}(\tau)+\varepsilon^{2} \mu_{2}^{\prime}(\tau)+\cdots\right) \delta \frac{\partial^{2} U}{\partial \hat{t} \partial \tau} \\
& +2 \delta \frac{\partial^{2} U}{\partial \hat{t} \partial \tau}+\delta \frac{\partial U}{\partial \hat{t}}\left(\varepsilon \mu_{1}^{\prime \prime}(\tau)+\varepsilon^{2} \mu_{2}^{\prime \prime}(\tau)+\varepsilon^{3} \mu_{3}^{\prime \prime}(\tau)+\cdots\right)+\delta^{2} \frac{\partial^{2} U}{\partial \tau^{2}}
\end{aligned}
$$

We have let

$$
U(x, \hat{t}, \tau ; \varepsilon \delta)=\sum_{i=1}^{\infty} \sum_{j=0}^{\infty} v^{i j}(x, \hat{t}, \tau ; \varepsilon \delta) \varepsilon^{i} \delta^{j}
$$

Substituting (13), (14) and (15) into (7), and equating coefficients of $\varepsilon^{i} \delta^{j}$ we get

$$
\begin{aligned}
& O(\varepsilon): \frac{\partial^{2} v^{10}}{\partial \hat{t}^{2}}+\frac{\partial^{4} v^{10}}{\partial x^{4}}+2 \lambda \frac{\partial^{2} v^{10}}{\partial x^{2}}+v^{10}=-2 F \frac{d^{2} \bar{U}}{d x^{2}} \\
& O(\varepsilon \delta): \frac{\partial^{2} v^{11}}{\partial \hat{t}^{2}}+\frac{\partial^{4} v^{11}}{\partial x^{4}}+2 \lambda \frac{\partial^{2} v^{11}}{\partial x^{2}}+v^{11}=-2 \frac{\partial^{2} v^{10}}{\partial \hat{t} \partial \tau}+\frac{\partial v^{10}}{\partial \hat{t}} \\
& O\left(\varepsilon \delta^{2}\right): \frac{\partial^{2} v^{12}}{\partial \hat{t}^{2}}+\frac{\partial^{4} v^{12}}{\partial x^{4}}+2 \lambda \frac{\partial^{2} v^{12}}{\partial x^{2}}+v^{12}=-2 \frac{\partial^{2} v^{11}}{\partial \hat{t} \partial \tau}+\frac{\partial v^{11}}{\partial \hat{t}}-\frac{\partial^{2} v^{10}}{\partial \tau^{2}} \\
& O\left(\varepsilon^{2}\right): \frac{\partial^{2} v^{20}}{\partial \hat{t}^{2}}+\frac{\partial^{4} v^{20}}{\partial x^{4}}+2 \lambda \frac{\partial^{2} v^{20}}{\partial x^{2}}+v^{20}=-2 \mu_{1}^{\prime} \frac{\partial^{2} v^{10}}{\partial \hat{t}^{2}}+\alpha\left(v^{10}\right)^{2} \\
& O\left(\varepsilon^{2} \delta\right): \frac{\partial^{2} v^{21}}{\partial \hat{t}^{2}}+\frac{\partial^{4} v^{21}}{\partial x^{4}}+2 \lambda \frac{\partial^{2} v^{21}}{\partial x^{2}}+v^{21}=-2 \mu_{1}^{\prime} \frac{\partial^{2} v^{11}}{\partial \hat{t}^{2}}-2 \mu_{1}^{\prime} \frac{\partial^{2} v^{10}}{\partial \hat{t} \partial \tau}-2 \frac{\partial^{2} v^{20}}{\partial \hat{t} \partial \tau}-\mu_{1}^{\prime \prime} \frac{\partial v^{10}}{\partial \hat{t}} \\
& +\frac{\partial v^{20}}{\partial \hat{t}}+\mu_{1}^{\prime} \frac{\partial v^{10}}{\partial \hat{t}}+2 v^{10} v^{11} \\
& O\left(\varepsilon^{2} \delta^{2}\right): \frac{\partial^{2} v^{22}}{\partial \hat{t}^{2}}+\frac{\partial^{4} v^{22}}{\partial x^{4}}+2 \lambda \frac{\partial^{2} v^{22}}{\partial x^{2}}+v^{22}=-2 \mu_{1}^{\prime} \frac{\partial^{2} v^{12}}{\partial \hat{t}^{2}}-2 \mu_{1}^{\prime} \frac{\partial^{2} v^{11}}{\partial \hat{t} \partial \tau}-2 \frac{\partial^{2} v^{21}}{\partial \hat{t} \partial \tau}-\mu_{1}^{\prime \prime} \frac{\partial v^{11}}{\partial \hat{t}} \\
& +\frac{\partial v^{21}}{\partial \hat{t}}+\mu_{1}^{\prime} \frac{\partial v^{11}}{\partial \hat{t}}+\frac{\partial v^{20}}{\partial \tau}+\alpha\left(\left(v^{11}\right)^{2}+2 v^{10} v^{12}\right) \\
& O\left(\varepsilon^{3}\right): \frac{\partial^{2} v^{30}}{\partial \hat{t}^{2}}+\frac{\partial^{4} v^{30}}{\partial x^{4}}+2 \lambda \frac{\partial^{2} v^{30}}{\partial x^{2}}+v^{30}=-\left(\mu_{1}^{\prime}\right)^{2} \frac{\partial^{2} v^{10}}{\partial \hat{t}^{2}}-2\left(\mu_{1}^{\prime} \frac{\partial^{2} v^{20}}{\partial \hat{t}^{2}}+\mu_{2}^{\prime} \frac{\partial^{2} v^{10}}{\partial \hat{t}^{2}}\right) \\
& +\alpha v^{10} v^{20}+\beta\left(v^{10}\right)^{3}
\end{aligned}
$$




$$
\begin{aligned}
& O\left(\varepsilon^{3} \delta\right): \frac{\partial^{2} v^{31}}{\partial \hat{t}^{2}}+\frac{\partial^{4} v^{31}}{\partial x^{4}}+2 \lambda \frac{\partial^{2} v^{31}}{\partial x^{2}}+v^{31}=-2\left(\mu_{1}^{\prime} \frac{\partial^{2} v^{21}}{\partial \hat{t}^{2}}+\mu_{2}^{\prime} \frac{\partial^{2} v^{11}}{\partial \hat{t}^{2}}\right) \\
&-\left(\mu_{1}^{\prime}\right)^{2} \frac{\partial^{2} v^{11}}{\partial \hat{t}^{2}}-2 \frac{\partial^{2} v^{30}}{\partial \hat{t} \partial \tau}-2\left(\mu_{1}^{\prime} \frac{\partial^{2} v^{20}}{\partial \hat{t} \partial \tau}+\mu_{2}^{\prime} \frac{\partial^{2} v^{10}}{\partial \hat{t} \partial \tau}\right) \\
&+\left(\frac{\partial v^{30}}{\partial \hat{t}}+\mu_{1}^{\prime} \frac{\partial v^{20}}{\partial \hat{t}}+\mu_{2}^{\prime} \frac{\partial v^{10}}{\partial \hat{t}}\right)+\alpha\left(v^{10} v^{21}+v^{20} v^{11}\right)+3 \beta\left(v^{10}\right)^{2} v^{11} \\
& O\left(\varepsilon^{3} \delta^{2}\right): \frac{\partial^{2} v^{32}}{\partial \hat{t}^{2}}+\frac{\partial^{4} v^{32}}{\partial x^{4}}+2 \lambda \frac{\partial^{2} v^{32}}{\partial x^{2}}+v^{32}=-\left(\mu_{1}^{\prime}\right)^{2} \frac{\partial^{2} v^{12}}{\partial \hat{t}^{2}}-\frac{\partial^{2} v^{30}}{\partial \tau^{2}} \\
&-2\left(\mu_{1}^{\prime} \frac{\partial^{2} v^{22}}{\partial \hat{t}^{2}}+\mu_{2}^{\prime} \frac{\partial^{2} v^{12}}{\partial \hat{t}^{2}}\right)-2\left(\mu_{1}^{\prime} \frac{\partial^{2} v^{21}}{\partial \hat{t} \partial \tau}+\mu_{2}^{\prime} \frac{\partial^{2} v^{11}}{\partial \hat{t} \partial \tau}\right)-2 \frac{\partial^{2} v^{31}}{\partial \hat{t} \partial \tau} \\
&-\left(\mu_{1}^{\prime \prime} \frac{\partial v^{21}}{\partial \hat{t}}+\mu_{2}^{\prime \prime} \frac{\partial v^{11}}{\partial \hat{t}}\right)+\left(\frac{\partial v^{31}}{\partial \hat{t}}+\mu_{1}^{\prime} \frac{\partial v^{21}}{\partial \hat{t}}+\mu_{2}^{\prime} \frac{\partial v^{11}}{\partial \hat{t}}+\frac{\partial v^{30}}{\partial \hat{t}}\right) \\
&+\alpha\left(v^{10} v^{22}+v^{11} v^{21}+v^{12} v^{20}\right)+\beta\left(3\left(v^{10}\right)^{2} v^{12}+v^{10}\left(v^{11}\right)^{2}\right)
\end{aligned}
$$

With the following initial conditions

$$
\begin{aligned}
& O(\varepsilon): \frac{\partial v^{10}(0,0)}{\partial \hat{t}}=0 \\
& O(\varepsilon \delta): \frac{\partial v^{11}(0,0)}{\partial \hat{t}}+\frac{\partial v^{10}(0,0)}{\partial \tau}=0 \\
& O\left(\varepsilon \delta^{2}\right): \frac{\partial v^{12}(0,0)}{\partial \hat{t}}+\frac{\partial v^{11}(0,0)}{\partial \tau}=0 \\
& O\left(\varepsilon^{2}\right): \frac{\partial v^{20}(0,0)}{\partial \hat{t}}+\mu_{1}^{\prime}(0) \frac{\partial v^{10}(0,0)}{\partial \hat{t}}=0 \\
& O\left(\varepsilon^{2} \delta\right): \frac{\partial v^{21}(0,0)}{\partial \hat{t}}+\mu_{1}^{\prime}(0) \frac{\partial v^{11}(0,0)}{\partial \hat{t}}+\frac{\partial v^{20}(0,0)}{\partial \tau}=0 \\
& O\left(\varepsilon^{2} \delta^{2}\right): \frac{\partial v^{22}(0,0)}{\partial \hat{t}}+\mu_{1}^{\prime}(0) \frac{\partial v^{12}(0,0)}{\partial \hat{t}}+\frac{\partial v^{21}(0,0)}{\partial \tau}=0 \\
& O\left(\varepsilon^{3}\right): \frac{\partial v^{30}(0,0)}{\partial \hat{t}}+\mu_{1}^{\prime}(0) \frac{\partial v^{20}(0,0)}{\partial \hat{t}}+\mu_{2}^{\prime}(0) \frac{\partial v^{10}(0,0)}{\partial \hat{t}}=0 \\
& O\left(\varepsilon^{3} \delta\right): \frac{\partial v^{31}(0,0)}{\partial \hat{t}}+\mu_{1}^{\prime}(0) \frac{\partial v^{21}(0,0)}{\partial \hat{t}}+\mu_{2}^{\prime}(0) \frac{\partial v^{11}(0,0)}{\partial \hat{t}}+\frac{\partial v^{30}(0,0)}{\partial \tau}=0 \\
& O\left(\varepsilon^{3} \delta^{2}\right): \frac{\partial v^{32}(0,0)}{\partial \hat{t}}+\mu_{1}^{\prime}(0) \frac{\partial v^{22}(0,0)}{\partial \hat{t}}+\mu_{2}^{\prime}(0) \frac{\partial v^{12}(0,0)}{\partial \hat{t}}+\frac{\partial v^{31}(0,0)}{\partial \tau}=0 .
\end{aligned}
$$

For solution, we assume

$v^{i j}(x, \hat{t}, \tau)=\sum_{n=1}^{\infty} v_{n}^{i j} \sin n x$

where

$\bar{U}=a_{m} \sin m x, m$ is fixed, $\left\|a_{m}\right\| \square 1$. 
We then solve the sequence of differential equations; on substituting (34) and (35) into (16), we have

$$
\sum_{n=1}^{\infty}\left[\frac{\partial^{2} v_{n}^{10}}{\partial \hat{t}^{2}}+\left(n^{4}-2 \lambda n^{2}+1\right) v_{n}^{10}\right] \sin n x=2 F m^{2} a_{m} \sin m x .
$$

Multiplying (36) by $\sin m x$ and integrating from $x=0$ to $x=\pi$, for $n=m$, we get

$$
\frac{\partial^{2} v_{m}^{10}}{\partial \hat{t}^{2}}+w_{m}^{2} v_{m}^{10}=2 F m^{2} a_{m}
$$

where

$$
w_{m}^{2}=n^{4}-2 \lambda n^{2}+1 .
$$

The solution of (37) is

$$
\begin{aligned}
& v_{m}^{10}(\hat{t}, \tau)=\alpha_{m}^{10}(\tau) \cos w_{m} \hat{t}+\beta_{m}^{10}(\tau) \sin w_{m} \hat{t}+B_{m}, \\
& B_{m}=\frac{2 F m^{2} a_{m}}{w_{m}^{2}} .
\end{aligned}
$$

Applying (25) on (39) we obtain

$v_{m}^{10}(0,0)=\alpha_{m}^{10}(0)+B_{m}=0$

$\alpha_{m}^{10}(0)=-B_{m}$

and

$\beta_{m}^{10}(0)=0$.

When $i=1$ and $j=1$, (34) becomes

$v^{11}(x, \hat{t}, \tau)=\sum_{n=1}^{\infty} v_{n}^{11}(\hat{t}, \tau) \sin n x$.

Substituting (43) into (24), we get

$\sum_{n=1}^{\infty}\left[\frac{\partial^{2} v_{n}^{11}}{\partial \hat{t}^{2}}+w_{n}^{2} v_{n}^{11}\right] \sin n x=\left(-2 \frac{\partial^{2} v_{m}^{10}}{\partial \hat{t}^{2}}+\frac{\partial v_{m}^{10}}{\partial \hat{t}}\right) \sin m x$.

Multiplying by $\sin m x$, integrating from $x=0$ to $x=\pi$ and dividing through by $\frac{\pi}{2}$, we obtain

$$
\begin{aligned}
\frac{\partial^{2} v_{n}^{11}}{\partial \hat{t}^{2}}+w_{n}^{2} v_{n}^{11}= & -2 \frac{\partial^{2} v_{m}^{10}}{\partial \hat{t}^{2}}+\frac{\partial v_{m}^{10}}{\partial \hat{t}} \\
& =-2\left(-\alpha_{m}^{10^{\prime}} \sin w_{m} \hat{t}+\beta_{m}^{10^{\prime}} \cos w_{m} \hat{t}\right)+\left(-\alpha_{m}^{10} \sin w_{m} \hat{t}+\beta_{m}^{10} \cos w_{m} \hat{t}\right) .
\end{aligned}
$$

Applying the solvability condition, we have

$2 \beta_{m}^{10^{\prime}}-\beta_{m}^{10}=0$

and

$2 \alpha_{m}^{10^{\prime}}-\alpha_{m}^{10}=0$.

The solution of (46) and (47) are given respectively

$\beta_{m}^{10}(\tau)=\beta_{m}^{10}(0) e^{\frac{\tau}{2}}=0$

and

$\alpha_{m}^{10}(\tau)=\alpha_{m}^{10}(0) e^{\frac{\tau}{2}}=-B_{m} e^{\frac{\tau}{2}}$.

Therefore 
$v_{m}^{10}(\hat{t}, \tau)=\alpha_{m}^{10}(0) \cos w_{m} \hat{t}+B_{m}$

and

$v^{10}(x, \hat{t}, \tau)=v_{m}^{10}(\hat{t}, \tau) \sin m x$.

The solution of the remaining equation in (45) is

$v_{m}^{11}(\hat{t}, \tau)=\alpha_{m}^{11}(\tau) \cos w_{m} \hat{t}+\beta_{m}^{11}(\tau) \sin w_{m} \hat{t}$.

Using the appropriate initial conditions we obtain

$$
\begin{aligned}
& \alpha_{m}^{11}(0)=0 \\
& \beta_{\mathrm{m}}^{11}(0)=-\frac{\alpha_{m}^{10^{\prime}}(0)}{w_{m}}=\frac{B_{m}}{w_{m}} .
\end{aligned}
$$

When $i=1$ and $j=2$, (34) become

$$
v^{12}(x, \hat{t}, \tau)=\sum_{n=1}^{\infty} v_{n}^{12}(\hat{t}, \tau) \sin n x .
$$

Substituting (55) into (18), we get

$$
\sum_{n=1}^{\infty}\left[\frac{\partial^{2} v_{n}^{12}}{\partial \hat{t}^{2}}+w_{n}^{2} v_{n}^{12}\right] \sin n x=\left(-2 \frac{\partial^{2} v_{m}^{11}}{\partial \hat{t} \partial \tau}+\frac{\partial v_{m}^{11}}{\partial \hat{t}}-\frac{\partial^{2} v_{m}^{10}}{\partial \hat{t} \partial \tau}\right) \sin m x
$$

Multiplying by $\sin m x$, integrating from $x=0$ to $x=\pi$ and dividing through by $\frac{\pi}{2}$, we obtain

$$
\begin{aligned}
\frac{\partial^{2} v_{n}^{12}}{\partial \hat{t}^{2}}+w_{n}^{2} v_{n}^{12}= & -2 \frac{\partial^{2} v_{m}^{11}}{\partial \hat{t} \partial \tau}+\frac{\partial v_{m}^{11}}{\partial \hat{t}}-\frac{\partial^{2} v_{m}^{10}}{\partial \hat{t} \partial \tau} \\
= & -2 w_{m}\left(-\alpha_{m}^{11 \prime} \sin w_{m} \hat{t}+\beta_{m}^{11^{\prime \prime}} \cos w_{m} \hat{t}\right) \\
& +w_{m}\left(-\alpha_{m}^{11} \sin w_{m} \hat{t}+\beta_{m}^{11} \cos w_{m} \hat{t}\right)-\alpha_{m}^{10^{\prime \prime}} \cos w_{m} \hat{t} .
\end{aligned}
$$

Using the secularity condition in (57), we get

$$
\begin{aligned}
& -2 \beta_{m}^{11^{\prime}}+\beta_{m}^{11}=\frac{\alpha_{m}^{10^{\prime \prime}}}{w_{m}}, \\
& -2 \alpha_{m}^{11^{\prime}}+\alpha_{m}^{11}=0 .
\end{aligned}
$$

The solution to (58) is

$$
\beta_{m}^{11}(\tau)=-e^{\frac{\tau}{2}}\left(\int_{0}^{\tau}\left(\frac{\alpha_{m}^{10^{\prime \prime}}}{w_{m}}\right) e^{s} d s+k_{1}\right),
$$




$$
\beta_{m}^{11}(\tau)=-e^{\frac{\tau}{2}}\left(\int_{0}^{\tau}\left(\frac{\alpha_{m}^{10^{\prime \prime}}}{w_{m}}\right) e^{s} d s-\frac{B_{m}}{w_{m}}\right)=-\frac{B_{m}}{w_{m}} e^{\frac{\tau}{2}}\left(\int_{0}^{\tau} e^{s} d s-1\right)
$$

Solution to (59) is

$$
\alpha_{m}^{11}(\tau)=\alpha_{m}^{11}(0) e^{\frac{\tau}{2}}=0
$$

Therefore

$$
v^{11}(x, \hat{t}, \tau)=v_{m}^{11}(\hat{t}, \tau) \sin m x
$$

The solution of the remaining equation in (57) is

$$
v_{m}^{12}(\hat{t}, \tau)=\alpha_{m}^{12}(\tau) \cos w_{m} \hat{t}+\beta_{m}^{12}(\tau) \sin w_{m} \hat{t} .
$$

Applying the proper initial condition we have

$$
\begin{aligned}
& \alpha_{m}^{12}(0)=0, \\
& \beta_{m}^{12}(0)=-\frac{\beta_{m}^{11^{\prime}}(0)}{w_{m}}=\frac{-2 B_{m}}{w_{m}^{2}} .
\end{aligned}
$$

Therefore

$$
v^{12}(x, \hat{t}, \tau)=v_{m}^{12}(\hat{t}, \tau) \sin m x
$$

Furthermore, substituting (34) into (19) when $i=2$ and $j=0$, we get

$$
\begin{aligned}
\sum_{n=1}^{\infty}\left[\frac{\partial^{2} v_{n}^{20}}{\partial \hat{t}^{2}}+w_{n}^{2} v_{n}^{20}\right] \sin n x & =-2 \mu_{1}^{\prime} \frac{\partial^{2} v_{m}^{10}}{\partial \hat{t}^{2}} \sin m x+\alpha\left(v_{m}^{10}\right)^{2} \sin ^{2} m x \\
& =-2 \mu_{1}^{\prime} \frac{\partial^{2} v_{m}^{10}}{\partial \hat{t}^{2}} \sin m x+\frac{\alpha}{2}\left(v_{m}^{10}\right)^{2}(1-\cos 2 m x)
\end{aligned}
$$

Multiplying (67) by $\sin m x$, integrating from $x=0$ to $x=\pi$, we obtain

$\frac{\pi}{2}\left[\frac{\partial^{2} v_{n}^{20}}{\partial \hat{t}^{2}}+w_{n}^{2} v_{n}^{20}\right]=-\pi \mu_{1}^{\prime} \frac{\partial^{2} v_{m}^{10}}{\partial \hat{t}^{2}}+\frac{\alpha}{2}\left(v_{m}^{10}\right)^{2} \int_{0}^{\pi}(1-\cos 2 m x) \sin m x d x$

On appropriate substitution of the value of $v_{m}^{10}$ in (69) and simplification, we have

$$
\begin{aligned}
\frac{\partial^{2} v_{n}^{20}}{\partial \hat{t}^{2}}+w_{n}^{2} v_{n}^{20}= & -2 \mu_{1}^{\prime}\left(w_{m}^{2} \alpha_{m}^{10} \cos w_{m} \hat{t}\right) \\
& +\frac{16 \alpha}{3 \pi m}\left[\left(\frac{\left(\alpha_{m}^{10}\right)^{2}}{2}+B_{m}\right)+\frac{\left(\alpha_{m}^{10}\right)^{2}}{2} \cos 2 w_{m} \hat{t}+2 B_{m} \alpha_{m}^{10} \cos w_{m} \hat{t}\right]
\end{aligned}
$$


To ensure uniformly valid asymptotic solution in $\hat{t}$, we equate to zero in (70) the coefficients of $\cos w_{m} \hat{t}$, we obtain

$$
\mu_{1}^{\prime}(\tau)=\frac{16 \alpha B_{m}}{3 m \pi w_{m}^{2}}
$$

The remaining equation in (70) is

$\frac{\partial^{2} v_{n}^{20}}{\partial \hat{t}^{2}}+w_{n}^{2} v_{n}^{20}=r_{0}+r_{1} \cos 2 w_{m} \hat{t}$

where

$r_{0}(\tau)=\frac{16 \alpha}{3 m \pi}\left(\frac{\left(\alpha_{m}^{10}(\tau)\right)^{2}}{2}+B_{m}^{2}\right)$
$r_{1}(\tau)=\frac{8 \alpha}{3 m \pi}\left(\alpha_{m}^{10}(\tau)\right)^{2}$.

The solution of $(72)$ is

$v_{m}^{20}(\hat{t}, \tau)=\alpha_{m}^{20}(\tau) \cos w_{m} \hat{t}+\beta_{m}^{20}(\tau) \sin w_{m} \hat{t}+\frac{r_{0}}{w_{m}^{2}}-\frac{r_{1} \cos 2 w_{m} \hat{t}}{3 w_{m}^{2}}$.

We substitute (34) into (20) when $i=2$ and $j=1$,we get

$$
\begin{aligned}
\sum_{n=1}^{\infty}\left[\frac{\partial^{2} v_{n}^{21}}{\partial \hat{t}^{2}}+w_{n}^{2} v_{n}^{21}\right] \sin n x & =\left(-2 \mu_{1}^{\prime} \frac{\partial^{2} v_{m}^{11}}{\partial \hat{t}^{2}}-2 \mu_{1}^{\prime} \frac{\partial^{2} v_{m}^{10}}{\partial \hat{t} \partial \tau}-\mu_{1}^{\prime \prime} \frac{\partial v_{m}^{10}}{\partial \hat{t}}-\mu_{1}^{\prime} \frac{\partial^{2} v_{m}^{20}}{\partial \hat{t} \partial \tau}+\frac{\partial v_{m}^{20}}{\partial \hat{t}}+2 \mu^{\prime} \frac{\partial v_{m}^{10}}{\partial \hat{t}}\right) \sin m x \\
& +2 \alpha v_{m}^{10} v_{m}^{11} \sin ^{2} m x
\end{aligned}
$$

Multiplying (76) $\sin m x$ and integrating from 0 to $\pi$, we get

$$
\begin{aligned}
\frac{\pi}{2}\left(\frac{\partial^{2} v_{n}^{21}}{\partial \hat{t}^{2}}+w_{n}^{2} v_{n}^{21}\right)=- & \frac{\pi}{2}\left(2 \mu_{1}^{\prime} \frac{\partial^{2} v_{m}^{11}}{\partial \hat{t}^{2}}+2 \mu_{1}^{\prime} \frac{\partial^{2} v_{m}^{10}}{\partial \hat{t} \partial \tau}+\mu_{1}^{\prime \prime} \frac{\partial v_{m}^{10}}{\partial \hat{t}}+\mu_{1}^{\prime} \frac{\partial^{2} v_{m}^{20}}{\partial \hat{t} \partial \tau}-\frac{\partial v_{m}^{20}}{\partial \hat{t}}-2 \mu^{\prime} \frac{\partial v_{m}^{10}}{\partial \hat{t}}\right) \\
& +2 \alpha \int_{0}^{\pi} v_{m}^{10} v_{m}^{11} \sin ^{3} m x d x .
\end{aligned}
$$

On appropriate substitution, we have

$$
\begin{aligned}
\frac{\partial^{2} v_{n}^{21}}{\partial \hat{t}^{2}}+w_{n}^{2} v_{n}^{21}=-\left(-2 \mu_{1}^{\prime} \beta_{m}^{11} w_{m}^{11} \sin w_{m} \hat{t}-2 w_{m} \alpha_{m}^{10^{\prime}} \sin w_{m} \hat{t}\right. \\
+2\left(-w_{m} \alpha_{m}^{20^{\prime}} \sin w_{m} \hat{t}+w_{m} \beta_{m}^{10^{\prime}} \cos w_{m} \hat{t}+\frac{2 w_{m} r_{1}^{\prime} \sin 2 w_{m} \hat{t}}{3 w_{m}^{2}}\right) \\
-w_{m} \alpha_{m}^{20} \sin w_{m} \hat{t}+w_{m} \beta_{m}^{20} \cos w_{m} \hat{t}+\frac{2 w_{m} r_{1} \sin 2 w_{m} \hat{t}}{3 w_{m}^{2}} \\
\left.-2 w_{m} \mu_{1}^{\prime} \alpha_{m}^{10} \sin w_{m} \hat{t}\right)+\frac{16 \alpha \beta_{m}^{11}}{3 m \pi}\left(\frac{\alpha_{m}^{10}}{2} \sin 2 w_{m} \hat{t}+B_{m} \sin w_{m} \hat{t}\right) .
\end{aligned}
$$

Using the so called secularity condition, we obtain that

$$
\beta_{m}^{20}(\tau) \equiv 0,
$$




$$
\alpha_{m}^{20}(\tau)=e^{\frac{\tau}{2}}\left(\int_{0}^{\tau} h_{1}(s) e^{s} d s+k_{2}\right)
$$

$$
h_{1}(\tau)=-2\left(\mu_{1}^{\prime} \beta_{m}^{11} w_{m}+\alpha_{m}^{10^{\prime}}+\mu_{1}^{\prime} \alpha_{m}^{10}+\frac{8 \alpha \beta_{m}^{11} B_{m}}{3 m \pi}\right) .
$$

The solution to the remaining equation in (78) is

$$
v_{m}^{21}(\hat{t}, \tau)=\alpha_{m}^{21}(\tau) \cos w_{m} \hat{t}+\beta_{m}^{21}(\tau) \sin w_{m} \hat{t}-\frac{r_{2} \sin 2 w_{m} \hat{t}}{3 w_{m}^{2}} .
$$

We substitute (34) into (21) when $i=2$ and $j=2$,we get

$$
\begin{gathered}
\sum_{n=1}^{\infty}\left(\frac{\partial^{2} v_{n}^{22}}{\partial \hat{t}^{2}}+w_{n}^{2} v_{n}^{22}\right) \sin n x=\left(2 \mu_{1}^{\prime} \frac{\partial^{2} v_{m}^{12}}{\partial \hat{t}^{2}}-2 \mu_{1}^{\prime} \frac{\partial^{2} v_{m}^{11}}{\partial \hat{t} \partial \tau}-\frac{\partial^{2} v_{m}^{21}}{\partial \hat{t} \partial \tau}-\mu_{1}^{\prime \prime} \frac{\partial v_{m}^{11}}{\partial \hat{t}}+\frac{\partial v_{m}^{21}}{\partial \hat{t}}+\mu_{1}^{\prime} \frac{\partial v_{m}^{11}}{\partial \hat{t}}+\frac{\partial v_{m}^{20}}{\partial \tau}\right) \sin m x \\
+\alpha\left(v_{m}^{11}\right)^{2} \sin ^{2} m x+2 \alpha v_{m}^{10} v_{m}^{12} \sin ^{2} m x
\end{gathered}
$$

Multiplying (83) by $\sin m x$, integrating from 0 to $\pi$, and making appropriate substitution we get

$$
\begin{aligned}
\frac{\partial^{2} v_{m}^{22}}{\partial \hat{t}^{2}}+w_{m}^{2} v_{m}^{22}= & -2 \mu_{1}^{\prime} \beta_{m}^{12} w_{m}^{2} \cos w_{m} \hat{t}-2 \mu_{1}^{\prime} \beta_{m}^{11^{\prime}} w_{m} \cos w_{m} \hat{t}+\alpha_{m}^{211^{\prime}} w_{m} \sin w_{m} \hat{t}-\beta_{m}^{21^{\prime}} w_{m} \cos w_{m} \hat{t} \\
& +\frac{2 w_{m} r_{2}^{\prime}}{3 w_{m}^{2}} \cos 2 w_{m} \hat{t}-\alpha_{m}^{21} w_{m} \sin w_{m} \hat{t}+\beta_{m}^{21} w_{m} \cos w_{m} \hat{t}-\frac{2 w_{m} r_{2}}{3 w_{m}^{2}} \cos 2 w_{m} \hat{t}+\mu_{1}^{\prime} \beta_{m}^{11} \cos w_{m} \hat{t} \\
& +\alpha_{m}^{20^{\prime}} \cos w_{m} \hat{t}+\beta_{m}^{20^{\prime}} \sin w_{m} \hat{t}+\frac{r_{0}^{\prime}}{w_{m}^{2}}-\frac{r_{1}^{\prime}}{3 w_{m}^{2}} \cos 2 w_{m} \hat{t} \\
& +\frac{8 \alpha}{3 m \pi}\left[\frac{\left(\beta_{m}^{11}\right)^{2}}{2}\left(1-\cos 2 w_{m} \hat{t}\right)+\frac{\beta_{m}^{12}}{2}\left(\alpha_{m}^{10} \cos 2 w_{m} \hat{t}+1\right)+\beta_{m}^{12} B_{m} \cos w_{m} \hat{t}\right] .
\end{aligned}
$$

Using the solvability condition on (84), we get

$$
\beta_{m}^{21}(\tau)=e^{-\tau}\left(\int_{0}^{\pi} h_{2}(s) e^{s} d s+k_{3}\right)
$$

and

$$
\alpha_{m}^{21}(\tau) \equiv 0
$$

The solution to the remaining equation in (84) is 
$v_{m}^{22}(\tau)=\alpha_{m}^{22} \cos w_{m} \hat{t}+\beta_{m}^{22} \sin w_{m} \hat{t}+\frac{r_{3}}{w_{m}^{2}}-\frac{r_{4}}{3 w_{m}^{2}} \cos 2 w_{m} \hat{t}$

and

$v^{22}(x, \hat{t}, \tau)=v_{m}^{22}(\hat{t}, \tau) \sin m x$.

We substitute (34) into (22) when $i=3$ and $j=0$,we get

$$
\begin{aligned}
\sum_{n=1}^{\infty}\left(\frac{\partial^{2} v_{n}^{30}}{\partial \hat{t}^{2}}+w_{n}^{2} v_{n}^{30}\right) \sin n x=( & \left.\left(\mu_{1}^{\prime}\right)^{2} \frac{\partial^{2} v_{m}^{10}}{\partial \hat{t}^{2}}-2 \mu_{1}^{\prime} \frac{\partial^{2} v_{m}^{20}}{\partial \hat{t}^{2}}-2 \mu_{2}^{\prime} \frac{\partial^{2} v_{m}^{10}}{\partial \hat{t}^{2}}\right) \sin m x \\
& +\frac{\alpha}{2} v_{m}^{10} v_{m}^{20}(1-\cos 2 m x)+\frac{\beta}{4}\left(v_{m}^{10}\right)^{3}(3 \sin m x-\sin 3 m x) .
\end{aligned}
$$

Multiplying through by $\sin m x$ at $n=m$, simplifying the coupling terms and integrating form 0 to $\pi$ we obtain

$$
\begin{aligned}
\frac{\partial^{2} v_{m}^{30}}{\partial \hat{t}^{2}}+w_{m}^{2} v_{m}^{30}= & \left(\mu_{1}^{\prime}\right)^{2} w_{m}^{2} \alpha_{m}^{10} \cos w_{m} \hat{t}-2 \mu_{1}^{\prime}\left(-\alpha_{m}^{20} w_{m}^{2} \cos w_{m} \hat{t}+w_{m}^{2} \beta_{m}^{20} \sin w_{m} \hat{t}-\frac{4 r_{1}}{3} \cos 2 w_{m} \hat{t}\right) \\
& +2 \mu_{2}^{\prime} w_{m}^{2} \alpha_{m}^{10} \cos w_{m} \hat{t}+\frac{3 \beta}{4}\left(\frac{\left(\alpha_{m}^{10}\right)^{3}}{4}\left(\cos 3 w_{m} \hat{t}+3 \cos w_{m} \hat{t}\right)+\frac{3\left(\alpha_{m}^{10}\right)^{2} B_{m}}{2}\left(\cos 2 w_{m} \hat{t}+1\right)\right) \\
& +\frac{8 \alpha}{3 m \pi}\left\{\frac{\alpha_{m}^{10} \alpha_{m}^{20}}{2} \cos 2 w_{m} \hat{t}+\frac{\alpha_{m}^{10} \beta_{m}^{20}}{2}+\frac{\alpha_{m}^{10} r_{0}}{w_{m}^{2}} \cos w_{m} \hat{t}-\frac{\alpha_{m}^{10} r_{1}}{6 w_{m}^{2}}\left(\cos 3 w_{m} \hat{t}+\cos w_{m} \hat{t}\right)\right. \\
& \left.+B_{m} \alpha_{m}^{10} \cos w_{m} \hat{t}+B_{m} \beta_{m}^{20} \sin w_{m} \hat{t}+\frac{B_{m} r_{0}}{w_{m}^{2}}-\frac{B_{m} r_{1}}{3 w_{m}^{2}} \cos 2 w_{m} \hat{t}\right\}
\end{aligned}
$$

To ensure uniformly valid asymptotic solution in $\hat{t}$, we equate to zero in (90) the coefficients of $\cos w_{m} \hat{t}$ and $\sin w_{m} \hat{t}$

$$
\therefore \mu_{2}^{\prime}(0)=\frac{\beta B_{m}^{2}}{\left(m \pi w_{m}^{2}\right)^{2}} S_{30}
$$

where

$$
s_{30}=\frac{\alpha^{2}}{\beta}\left\{\frac{32}{9 w_{m}}-\left(\frac{16}{27}+\frac{9}{4}\left(m \pi w_{m}\right)^{2}\right)\right\} \text {. }
$$

The solution of the remaining equation in (90) is

$v_{m}^{30}(\hat{t}, \tau)=\alpha_{m}^{30} \cos w_{m} \hat{t}+\beta_{m}^{30} \sin w_{m} \hat{t}+\frac{r_{5}}{w_{m}^{2}}-\frac{r_{6} \cos 2 w_{m} \hat{t}}{3 w_{m}^{2}}-\frac{r_{7} \cos 3 w_{m} \hat{t}}{8 w_{m}^{2}}$.

Thus the emotional state $u(x, \hat{t}, \tau)$ becomes

$u(x, \hat{t}, \tau)=\varepsilon\left(v_{m}^{10} \sin m x+\delta v_{m}^{11} \sin m x+\delta^{2} v_{m}^{12} \sin m x+\cdots\right)+\varepsilon^{2}\left(v_{m}^{20} \sin m x+\delta v_{m}^{21} \sin m x\right.$

$\left.+\delta^{2} v_{m}^{12} \sin m x+\cdots\right)+\varepsilon^{3}\left(v_{m}^{30} \sin m x+v_{3 m}^{30} \sin m x+\delta\left(v_{m}^{31} \sin m x++v_{3 m}^{31} \sin m x\right)+\cdots\right)+\cdots$ 


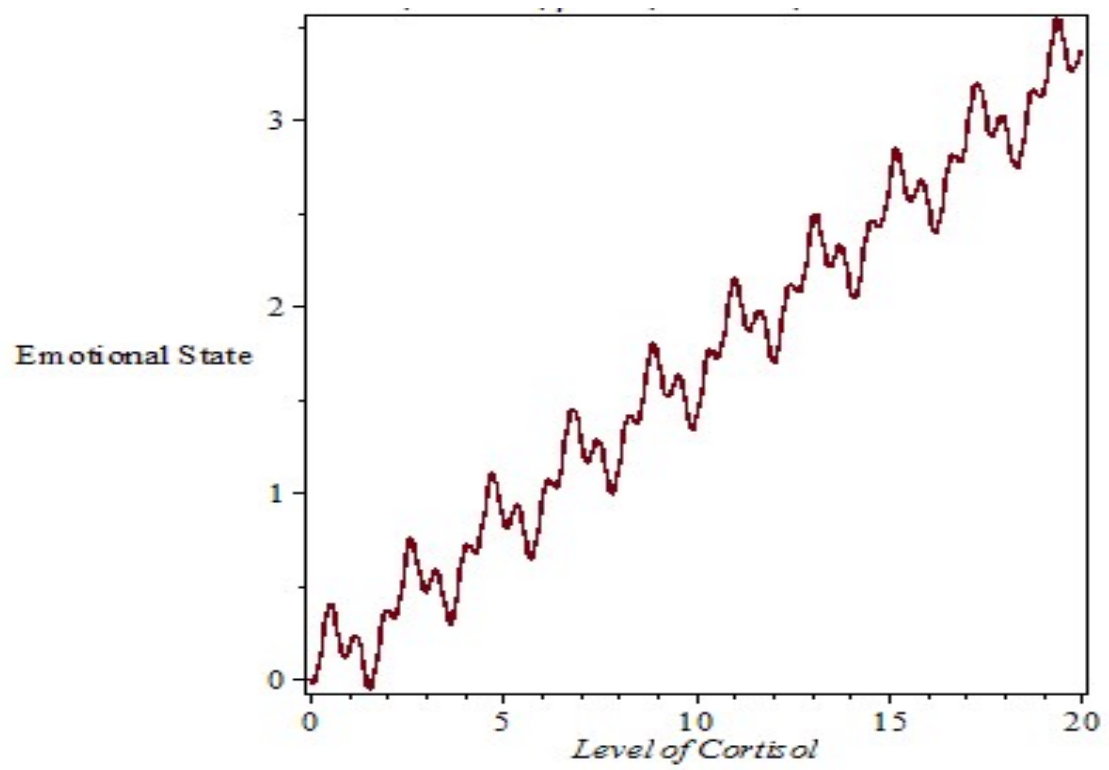

Fig. 1: Mood variation and level of cortisol in the blood $(\mu \mathrm{gm} / 100 \mathrm{ml})$, when $\delta=0.01, \alpha=-30, \beta=40$, $F=0.50, \lambda=0.43$.

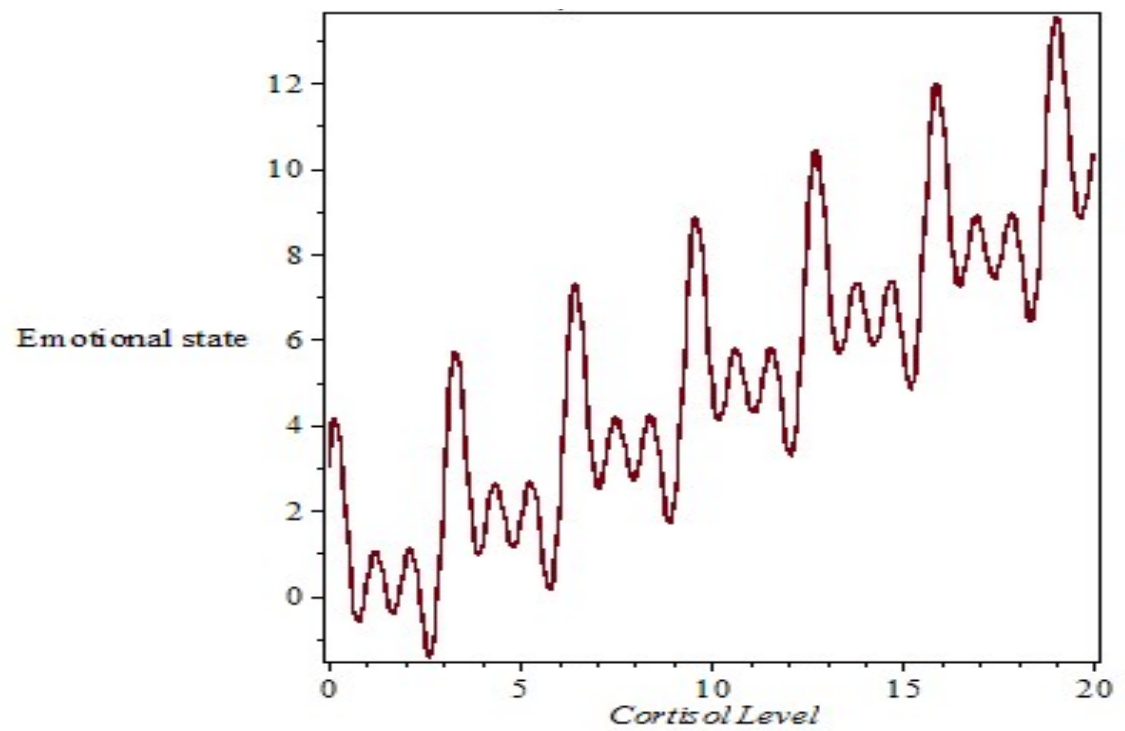

Fig. 2: Mood variation and level of cortisol in the blood $(\mu \mathrm{gm} / 100 \mathrm{ml})$, when $\delta=0.05, \alpha=30, \beta=-40$, $F=0.50, \lambda=0.5$.

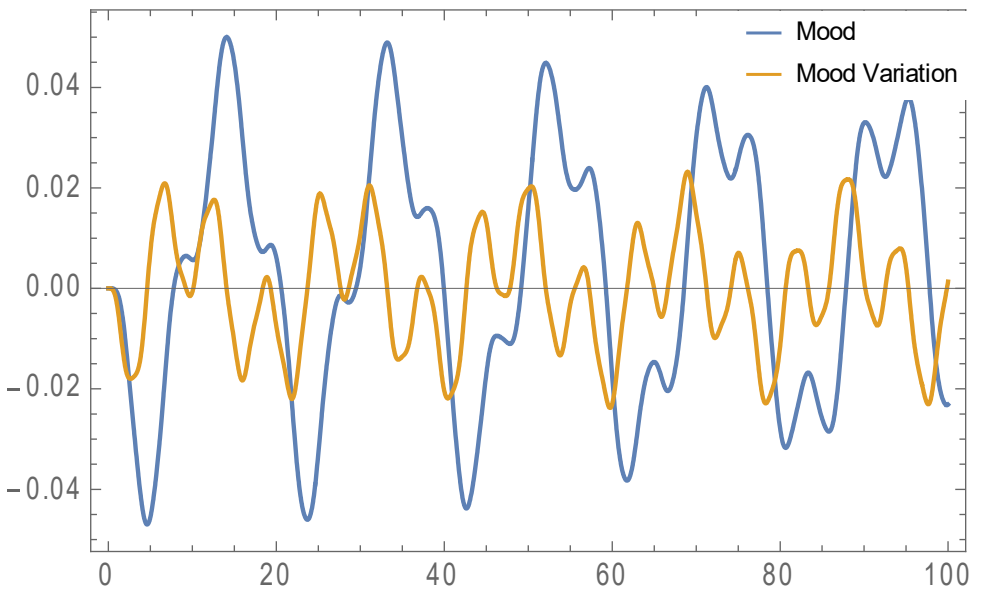

Fig. 3: Mood variation versus mood and level of cortisol in the blood $(\mu \mathrm{gm} / 100 \mathrm{ml})$, when $\delta=0.05$, $\alpha=30, \beta=-40, F=0.50, \lambda=0.5$. 


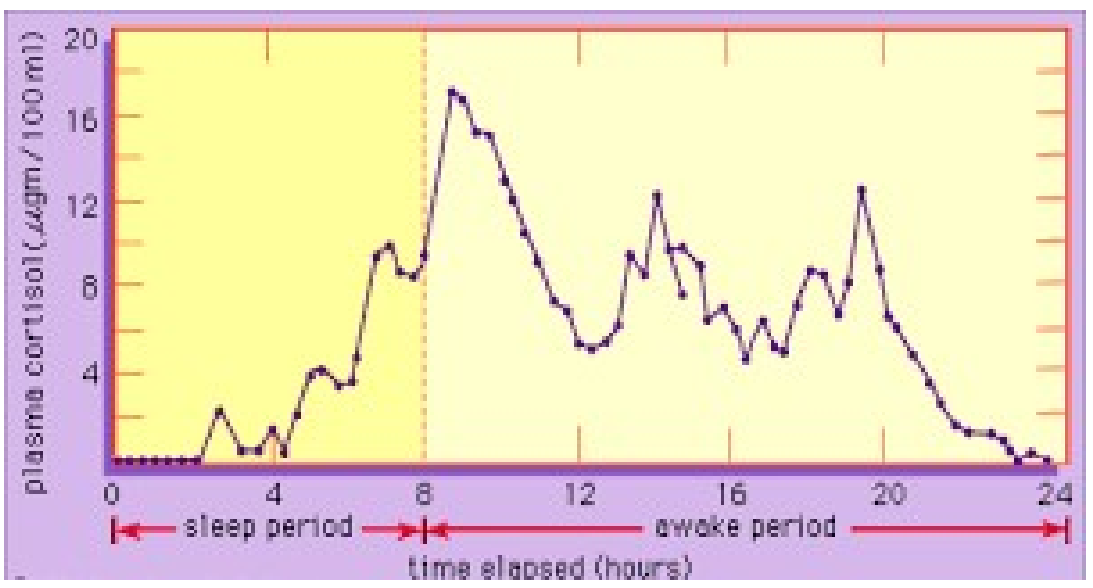

Fig. 4: Circadian rhythm of Plasma cortisol in the blood $(\mu \mathrm{gm} / 100 \mathrm{ml})$ for normal human adopted from Encyclopedia Britannica 2018.

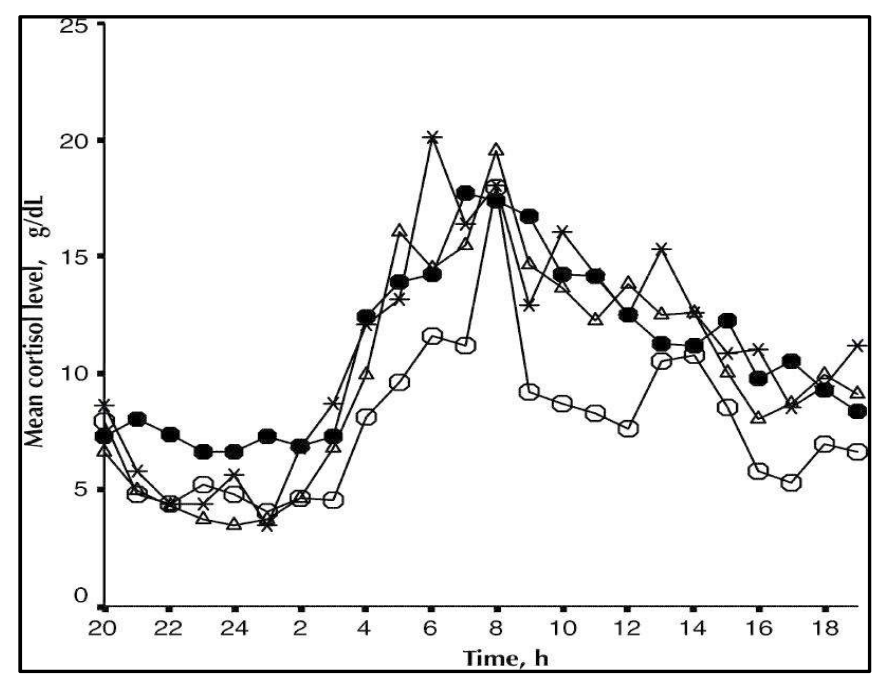

Fig. 5: Mean 24-h cortisol secretion of normal controls (open circles,) and patients with bipolar disorder in depressed (black circles), hypomanic (asterisks) and euthymic (open triangles) phases of their illness.

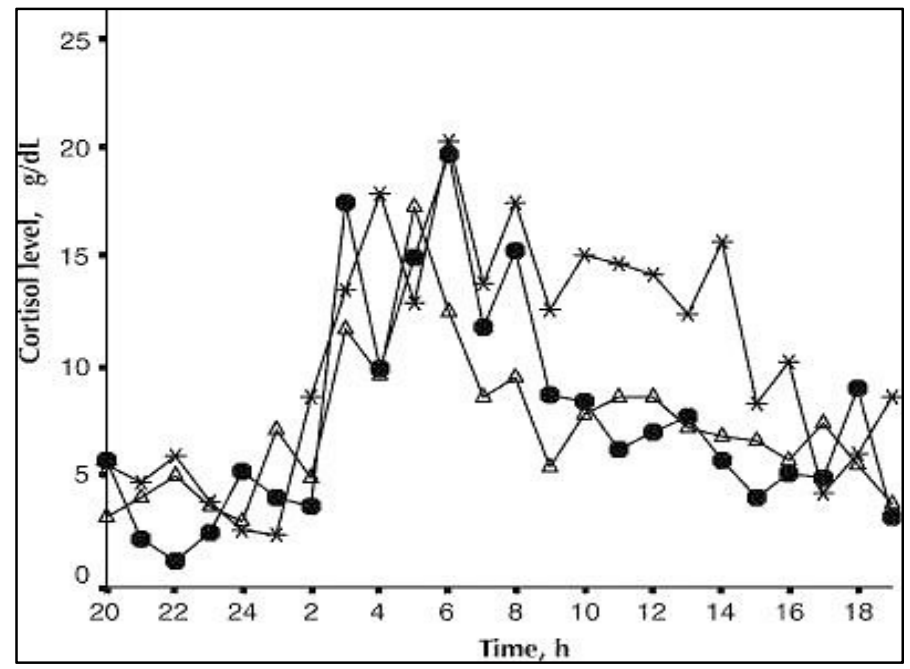

Fig. 6: The 24-h cortisol secretion profile of Ms. G.C. in 3 mood phases of bipolar disorder. Black circles, depressed phase; triangles, euthymic phase; asterisks, hypomanic phase. 


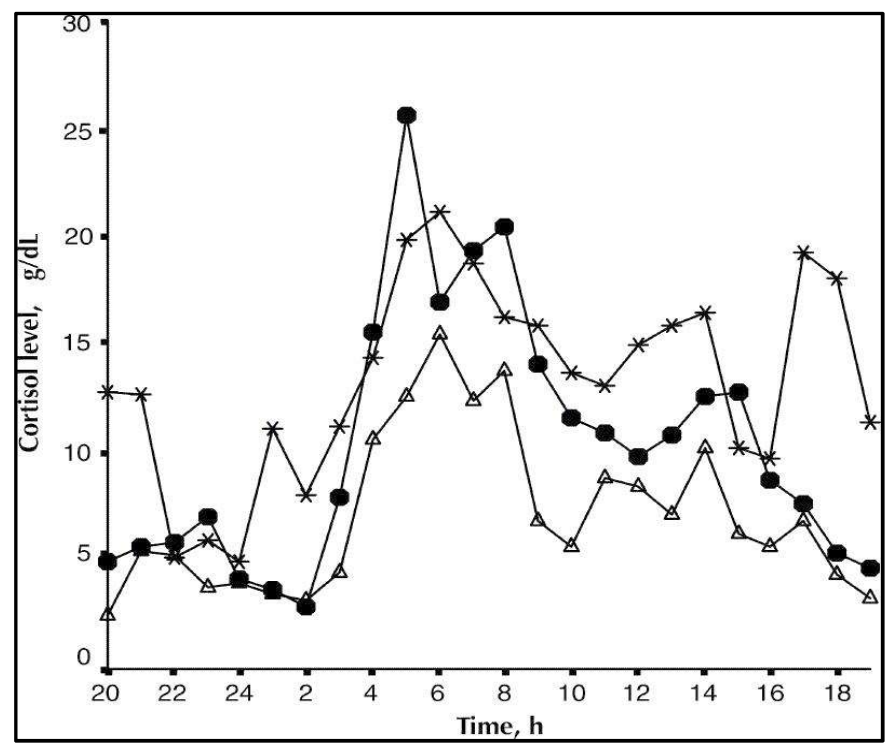

Fig. 7: The 24-h cortisol secretion profile of Mr. J.B. in 3 mood phases of bipolar disorder. Black circles, depressed phase; triangles, euthymic phase; asterisks, hypomanic phase.

\subsection{Discussion}

Cortisol is the main byproduct of HPA axis activities. Stress situations trigger the HPA axis, which is essential in raising the concentration of cortisol that leads to release of energy to the organism. Stress, both acute and chronic, has been identified as an essential etiological factor of depression. Fig. 1 and Fig. 2 show that hypercortisolism and hypocortisolism have significant effect on the mood variation of bipolar II disorder patient. HPA axis hyperactivity or hypoactivity can be termed as HPA axis dysregulation that might partly mediate the increased risk of BD relapse following intense psychosocial stress [18]. HPA axis hyperactivity predicted clinical relapses [19] and hypoactivity is associated with a higher number of mood episodes [20]. [21] explains that relative hypercortisolism were common among younger and more often females while hypocortisolism is common among older patients; must often inherent in patients that was not on Lithium. Since hypercortisolism is more peculiar among younger BD patients, we can suggest that hypocortisolism results from accumulated stress load from physiology, environmental and severe ailment episodes. This view is buttressed by [21,22] with studies performed on stress - related disorder and general population samples that collectively point at increased chronic stress and increased accumulated stress load as central to the development of the phenomenon.

Fig. 3 relates the mood variation and mood as the circadian rhythm of the cortisol is regulated. The observation shows that there is no significant change but the mood and its variation responds to the physiological reactions and environmental pulls. Fig. 4 is a typical circadian rhythm of plasma cortisol regulations in the blood stream for a normal human; hence, the oscillation buttress the indication in Fig. 1,2 and 3. Fig. $5-7$ represent cortisol secretion profile for the different episode of bipolar II disorder patients. They show that there are no significant circadian 
phase shifts associated with the of ailment. The increase and decrease in cortisol secretion in BD patients in both depressed and hypomanic phases suggest that cortisol level is not emblematic of bipolar II disorder but should not be relegated.

BD has a multifactorial etiology, depending on both genetic and environmental factors. Lately [23] estimated that monozygotic twin show concordance between 40 and $70 \%$, and a heritabitity of around $90 \%$. Enormous research has been conducted on the genetic risk factors of BD [24], genes related to HPA axis activity were not found to be significant risk factors for BD. Common polymorphisms of HPA - related genes are associated with different clinical features of BD. Variants of "clock" genes confer a vulnerability to circadian rhythms instability and to BD itself [25]. Environmental stressors such as parental neglect can lead to a vertical, intergenerational transmission of HPA axis abnormalities [21]; suggested that it might occur as a result of childhood trauma and dysregulated parenting style [20]. Recently [26] showed that childhood traumatic experiences can determine epigenetic modifications of the glucocortisolic receptors (GR) gene in patients with BD, HPA axis dysfunction might act as a mediator, on the basis of gene - environmental interactions [27]. Summarily, HPA axis hyperactivity or hypoactivity can be considered as an endophenotype, i.e. an etiological factor of BD but rather as a pathogenetic mechanism that can contribute to shape the clinical presentation of the disorder, on the ground of genetic - environmental interplay.

\subsection{Conclusion}

Bipolar II disorder and HPA axis activities are highly related. HPA axis hyperactivity in young folks and hypoactivity in older folks are associated with significant capacity to the hypomanic episode, likewise the depressive episode; as a result HPA abnormalities will partly affect BD patient. Numereous evidence suggests that HPA axis abnormalities should not be considered as an etiological or endophenotype factor of BD but as a pathogenetic and pathophysiological mechanism that contributes to shape BD clinical presentation $[24,28]$. Conclusively, HPA axis might be a great target pharmacologically to improvement of bipolar II disorder patients regardless that there are no significant circadian phase shifts in the various episodes.

\section{REFERENCES}

[1] G.Chrousos, (1998), Editorial: Ultradian, Circadian and stress - related hypothalamic - pituitary - adrenal axis activity - a dynamic digital - to - analog modulation. Endocrinology 139, 437.

[2] Vladimir M. M. , Cupic Z., Vladana V. and Kolar - Anic L., (2011); Predictive modeling of the hypothalamic - pituitary - adrenal (HPA) axis response to acute and chronic stress: Endocrine Journal Advance Ej11 - 0037.

[3] Miller W. L., Chrousos G. P., (2001); The Adrenal cortex. In Felig P, Frohman L (eds) Endocrinology and metabolism. McGraw - Hill, New York: 387 - 524.

[4] Tsigos C., Chrousos G. P. (2002); Hypothalamic - Pituitary Adrenal axis, neuroendocrine factors and stress. J Psychsom Res 53: $865-871$. 
[5] Stavreva D. A., Wiench M., John S., Couway - Campbell B. L., McKenna M. A., Pooley J. R., Johnson T. A., Vess T. C., Lightman S. L., Hager G. L., (2009); Ultradian hormone stimulation induces glucocorticoid receptor - mediated pulses of gene transcription. Nat cell Biol 11: 1093 1102.

[6] Russel G. M., Henley D.E., Leendertz J., Douthwaite J. A., Wood S. A., Stevens A, Woltersdorf W. W., Peeters B. W., Ruigt G. S., White A., Veldhius J. D., Lightman S. L., (2010); Rapid glucocorticoid receptor - mediated inhibition of hypothalamic - pituitary - adrenal ultradian activity in healthy males. J Neurosci 30: 6106-6115.

[7] Jeffrey G., Tasker and James P. H., (2011), Mechanism of rapid glucocorticoid feedback inhibition of the hypothalamic - pituitary - adrenal axis. Informa Healthcare 14(4): 398 - 406.

[8] Jelic S., Cupic Z., Kolar - Anic L. J., (2008), Modeling of the hypothalamic - pituitary - adrenal System activity based on the stoichiometric analysis. In Romano E, De Luca S (eds) New research on neurosecretory systems. Nova Science Publishers Inc. New York: 225 - 245.

[9] Walker J. J., Terry J. R., Lightman S. L., (2010), Origin of Ultradian pulsatility in the hypothalamic - pituitary - adrenal axis. Porc R Soc B 277: 1627 - 1633.

[10] Markovic V., Jelic S., Vukojevic V., Kolar Anic L. J. (2010), Modelling the hypothalamicpituitary - adrenal axis response to external perturbations with cortisol. Physical Chemistry 2010. Society of physical chemists of Serbia, Belgrade: $212-214$.

[11] Hosseinichimch, Nujousha, Rahmandad, Hazhir and Wittenbom, Andrea K. (2015), Modeling the hypothalamic - pituitary - adrenal axis: A review and extension. Mathematical Biosciences 268: $52-65$.

[12] Dickmics T., Weger B. D., Weger M., (2013), The circadian clock and glucocorticoids interactions across many time scales. Molecular and cellular endocrinology $380(1-2): 2-15$.

[13] Albrecht U., (2012), Timing to perfection: The biology of central and peripheral circadian clocks. Neuron 74(2): $246-260$.

[14] Bornstein S. R., England W.C., Ehrhart B. M., Herman J. P., (2008), Dissociation of ACTH and glucocorticoids. Trends in Endocrinology and metabolism TEM 19(5): 175180.

[15] Vanltallic T. B., (2002), Stress: a risk factor for serious illness. Metabolism 51: 40 - 45.

[16] Belmaker R.H., Agam G., (2008), Major depressive disorder. The new England Journal of Medicine 358(1): $47-60$.

[17] Rand R. H., Armbruser J. (1985), Perturbation methods, bifurcation theory and computer algebra. New York: Springer Verlag.

[18] Weiss, R.B., Stange, J.P., Boland, E.M., Black, S.K., LaBelle, D.R., Abramson, L.Y., Alloy,L.B., (2015). Kindling of life stress in bipolar disorder: comparison of sensitization and autonomy models. J. Abnorm. Psychol. 124, 4-16.

[19] Havermans, R., Nicolson, N.A., Berkhof, J., Devries, M.W., (2011). Patterns of salivary cortisol secretion and responses to daily events in patients with remitted bipolar disorder.

Psychoneuroendocrinology 36 (2), 258-265. 
[20] Ellenbogen, M.A., Linnen, A.M., Santo, J.B., aan het Rot, M., Hodgins, S., Young, S.N.,(2013). Salivary cortisol and interpersonal functioning: an event-contingent recording study in the offspring of parents with bipolar disorder. Psychoneuroendocrinology 38, 997-1006.

[21] Maripuu, M., Wikgren, M., Karling, P., Adolfsson, R., Norrback, K.F., (2017). Hyper- and hypocortisolism in bipolar disorder - A beneficial influence of lithium on the HPA-axis? J. Affect. Disord. 204, 187-196.

[22] Gustafsson, P.E., Janlert, U., Virtanen, P., Hammarstrom, A., (2012). The association between long-term accumulation of temporary employment, the cortisol awakening response and circadian cortisol levels. Psychoneuroendocrinology 37, 789-800.

[23] Craddock, N., Sklar, P., (2013). Genetics of bipolar disorder. Lancet 381,1654-1662.

[24] Belvederi Murri, M., Pariante, C., Mondelli, V., Masotti, M., Atti, A.R., Mellacqua, Z.,Antonioli, M., Ghio, L., Menchetti, M., Zanetidou, S., Innamorati, M., Amore, M.,(2016). HPA axis and aging in depression: systematic review and meta-analysis. Psychoneuroendocrinology 41, 46-62.

[25] Gonzalez, R., (2014). The relationship between bipolar disorder and biological rhythms. J. Clin. Psychiatry 75, e323-e331.

[26] Perroud, N., Dayer, A., Piguet, C., Nallet, A., Favre, S., Malafosse, A., Aubry, J.M.,(2014). Childhood maltreatment and methylation of the glucocorticoid receptor gene NR3C1 in bipolar disorder. Br. J. Psychiatry 204, 30-35.

[27] Ostiguy, C.S., Ellenbogen, M.A., Walker, C.D., Walker, E.F., Hodgins, S., (2011).Sensitivity to stress among the offspring of parents with bipolar disorder: a study of daytime cortisol levels. Psychol. Med. 41 (11), 2447-2457.

[28] Watson, S., Gallagher, P., Porter, R.J., Smith, M.S., Herron, L.J., Bulmer, S., Young,A.H., Ferrier, I.N., (2012). A randomized trial to examine the effect ofmifepristone on neuropsychological performance and mood in patients with bipolar depression. Biol. Psychiatry 72 (11), 943-949. 